\title{
Measurement of the decoherence function with the MACRO detector at Gran Sasso
}

\author{
S. Ahlen, ${ }^{\mathrm{c}}$ M. Ambrosio, ${ }^{1}$ R. Antolini, ${ }^{\mathrm{b}}$ G. Auriemma, ${ }^{\mathrm{n} *}$ A. Baldini, ${ }^{\mathrm{m}}$ G. C. Barbarino, ${ }^{1}$ \\ B. C. Barish, ${ }^{d}$ G. Battistoni, ${ }^{f}{ }^{\dagger}$ R. Bellotti, ${ }^{a}$ C. Bemporad,${ }^{m}$ P. Bernardini, ${ }^{j}$ H. Bilokon, ${ }^{f}$ V. Bisi, ${ }^{p}$ \\ C. Bloise,${ }^{f}$ S. Bussino, ${ }^{n}$ F. Cafagna,${ }^{a}$ M. Calicchio, ${ }^{a}$ D. Campana, ${ }^{1}$ P. Campana,${ }^{f}$ M. Carboni ${ }^{f}$ \\ S. Cecchini, ${ }^{b, \ddagger}$ F. Cei,${ }^{m}$ V. Chiarella,${ }^{f}$ C. Chiera,${ }^{n}$ A. Cobis, ${ }^{f}$ R. Cormack, ${ }^{c}$ A. Corona,${ }^{n}$ \\ S. Coutu, ${ }^{\mathrm{d}}$ G. DeCataldo, ${ }^{\mathrm{a}}$ H. Dekhussi, ${ }^{\mathrm{b}}$ C. DeMarzo, ${ }^{\mathrm{a}}$ M. De Vincenzi, ${ }^{\mathrm{n}}$ A. Di Credico, ${ }^{\mathrm{i}}$ E. Diehl, ${ }^{\mathrm{k}}$ \\ O. Erriquez, ${ }^{a}$ C. Favuzzi, ${ }^{a}$ D. Ficenec,,${ }^{c}{ }^{\S}$ C. Forti, ${ }^{f}$ L. Foti, ${ }^{n}$ P. Fusco, ${ }^{a}$ G. Giacomelli, ${ }^{b}$ \\ G. Giannini, ${ }^{\mathrm{m}, * *}$ N. Giglietto, ${ }^{\mathrm{a}}$ P. Giubellino, ${ }^{\mathrm{P}}$ M. Grassi, ${ }^{\mathrm{m}}$ P. Green, ${ }^{\mathrm{r}}$ A. Grillo, ${ }^{\mathrm{f}} \mathrm{F}$. Guarino,

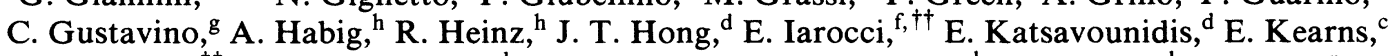 \\ S. Klein, ${ }^{c, \neq \ddagger}$ S. Kyriazopoulou, ${ }^{d}$ E. Lamanna, ${ }^{n}$ C. Lane, ${ }^{e}$ C. Lee, ${ }^{k}$ D. S. Levin, ${ }^{k}$ P. Lipari, ${ }^{n}$

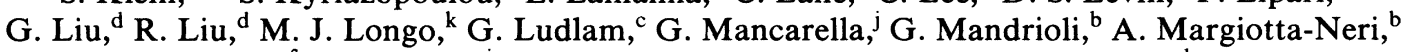 \\ A. Marin, ${ }^{\text {c }}$ A. Marini, ${ }^{f}$ D. Martello, ${ }^{\text {j }}$ A. Marzari Chiesa, ${ }^{\mathrm{p}}$ M. Masera, ${ }^{\mathrm{p}}$ P. Matteuzzi, ${ }^{\mathrm{b}}$ D. G. Michael, ${ }^{\mathrm{d}}$

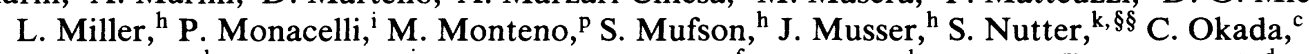 \\ G. Osteria, ${ }^{1}$ O. Palamara ${ }^{\mathrm{j}}$ S. Parlati, ${ }^{\mathrm{g}}$ V. Patera, ${ }^{\mathrm{f}}$ L. Patrizii, ${ }^{\mathrm{b}}$ R. Pazzi, ${ }^{\mathrm{m}}$ C. W. Peck, ${ }^{\mathrm{d}}$ \\ J. Petrakis, ${ }^{\mathrm{q}}$ S. Petrera, ${ }^{\mathrm{j}}$ N. D. Pignatano, ${ }^{\mathrm{d}}$ P. Pistilli, ${ }^{\mathrm{j}}$ F. Predieri, ${ }^{\mathrm{b}}{ }^{\mathrm{L}}$. Ramello, ${ }^{\mathrm{p}}{ }^{\mathrm{d}}$. Reynoldson ${ }^{\mathrm{g}}$ \\ F. Ronga, ${ }^{f}$ G. Rosa, ${ }^{\mathrm{n}}$ C. Satriano, ${ }^{\mathrm{n}, *}$ L. Satta,${ }^{\mathrm{f}+\dagger}$ E. Scapparone, ${ }^{\mathrm{b}}$ K. Scholberg, ${ }^{\mathrm{d}}$ A. Sciubba, ${ }^{\mathrm{n},{ }^{+\dagger}}$ \\ P. Serra Lugaresi, ${ }^{b}$ M. Severi, ${ }^{\mathrm{n}}$ M. Sitta, ${ }^{\mathrm{p}}$ P. Spinelli, ${ }^{\mathrm{a}}$ M. Spinetti, ${ }^{\mathrm{f}}$ M. Spurio, ${ }^{\mathrm{b}}$ J. Steele, ${ }^{\mathrm{d}}$ \\ R. Steinberg, ${ }^{\text {J J. L. Stone, }}{ }^{\mathrm{c}}$ L. R. Sulak, ${ }^{\mathrm{c}}$ A. Surdo, ${ }^{\mathrm{j}}$ G. Tarlé, ${ }^{\mathrm{k}}$ V. Togo, ${ }^{\mathrm{b}}$ V. Valente, ${ }^{\mathrm{f}}$ \\ C. W. Walter, ${ }^{d}$ R. Webb, ${ }^{\circ}$ and W. Worstell ${ }^{\mathrm{c}}$

\section{(MACRO Collaboration)}

\begin{abstract}
${ }^{a}$ Dipartimento di Fisica dell'Universitá di Bari and Istituto Nazionale di Fisica Nucleare, Bari 70126, Italy ${ }^{\mathrm{c}}$ Physics Department, Boston University, Boston, Massachusetts 02215 ${ }^{\mathrm{d} C a l i f o r n i a}$ Institute of Technology, Pasadena, California 91125

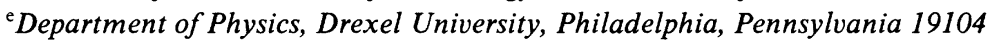

${ }^{\mathrm{f}}$ Laboratori Nazionali di Frascati dell' Istituto Nazionale di Fisica Nucleare, Frascati, Roma 00044, Italy ${ }^{\mathrm{g}}$ Laboratori Nazionali del Gran Sasso dell' Istituto Nazionale di Fisica Nucleare, Assergi (L'Aquila) 67010, Italy

${ }^{\mathrm{h}}$ Department of Physics and Department of Astronomy, Indiana University, Bloomington, Indiana 47405

${ }^{j}$ Dipartimento di Fisica dell'Universitá di Lecce and Istituto Nazionale di Fisica Nucleare, Lecce 73100, Italy

${ }^{k}$ Department of Physics, University of Michigan, Ann Arbor, Michigan 48109

'Dipartimento di Fisica dell'Universitá di Napoli and Istituto Nazionale di Fisica Nucleare, Napoli 80125, Italy

${ }^{\mathrm{m}}$ Dipartimento di Fisica dell'Universitá di Pisa and Istituto Nazionale di Fisica Nucleare, Pisa 56010, Italy

${ }^{\mathrm{n}}$ Dipartimento di Fisica dell'Universitá di Roma and Istituto Nazionale di Fisica Nucleare, Roma 00185, Italy ${ }^{\circ}$ Physics Department, Texas A\&M University, College Station, Texas 77843

${ }^{\mathrm{P}}$ Dipartimento di Fisica dell'Universitá di Torino and Istituto Nazionale di Fisica Nucleare, Torino 10125, Italy ${ }^{9}$ Bartol Research Institute, University of Delaware, Newark, Delaware 19716 'Sandia National Laboratory, Albuquerque, New Mexico 87185
\end{abstract}

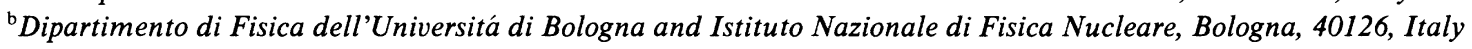 \\ 'Dipartimento di Fisica dell'Universitá dell'Aquila and Istituto Nazionale di Fisica Nucleare, L'Aquila 67100, Italy
}

(Received 26 May 1992)

\begin{abstract}
A measurement of the underground muon decoherence function has been performed using the multiple muon events collected by the MACRO detector at the Gran Sasso National Laboratory. A detector-independent analysis is presented for different zenith regions and rock depths; this allows direct comparison with any model of hadronic interactions. The measured decoherence function is compared with the predictions of a Monte Carlo simulation based on data taken by recent collider experiments.
\end{abstract}

PACS number(s): 96.40.Tv, 96.40.De

\footnotetext{
*Also università della Basilicata, Potenza 85100, Italy.

†Now at INFN Milano, Italy.

$\ddagger$ Also at Istituto TESRE/CNR, Bologna, Italy.

$\S_{\text {Now at }}$ Physics Department, Washington University, St Louis, MO 63130.

**Also at Universitá di Trieste and INFN, Trieste 34100, Italy.
}

\footnotetext{
††Also at Dipartimento di Energetica, Universitá di Roma, Roma 00185, Italy.

$\ddagger$ Now at Department of Physics, University of California, Santa Cruz, CA 95064.

$\S \S$ Currently at Laboratori Nazionali del Gran Sasso dell'INFN, 67010 , Italy.
} 


\section{INTRODUCTION}

The lateral separation and multiplicity of deep underground muon bundles are sensitive to the chemical composition and energy spectra of the primary cosmic rays incident in the Earth's atmosphere, to their initial interaction and to the ensuing hadronic cascade [1]. In this paper we report on an analysis of multiple-muon events observed during 7200 live hours of operation of the MACRO detector at the Gran Sasso National Laboratory. We specifically address aspects of the hadronic interaction through a comparison of the decoherence function derived from our data with that expected from Monte Carlo simulations based on recent results from collider experiments. The sensitivity of multiple muon rates to the cosmic-ray chemical composition is the focus of a companion paper [2].

In the remainder of this section, we define the decoherence function and outline the physics contributing to its evolution. Subsequent sections are devoted to the Monte Carlo simulation (Sec. II), the description of the MACRO detector (Sec. III), data-selection criteria (Sec. IV), the detector-independent data analysis methods (Sec. V), and the comparison of the data with the Monte Carlo simulation (Sec. VI). Conclusions are given in Sec. VII.

Deep underground muons observed in MACRO (average depth $3700 \mathrm{hg} / \mathrm{cm}^{2}$ of standard rock; minimum depth $3200 \mathrm{hg} / \mathrm{cm}^{2}$ ) are the decay products of high-energy charged mesons, essentially pions and kaons, produced by primary cosmic rays in the early stages of the hadronic cascade in the atmosphere. The minimum primary energy $\left(E_{\min }\right)$ required to produce a single muon at the detector depth is $\sim 3 \mathrm{TeV}$, while for two or more muons (the sample considered in this analysis) $E_{\min } \sim 20 \mathrm{TeV}$. These muons belong mostly to the kinematic region of high rapidity (fragmentation region) [1], which is not easily accessed by collider experiments.

In the laboratory frame, muons propagate through the atmosphere in a narrow cone whose opening angle with respect to the incident cosmic-ray direction is determined by the energy and transverse momentum $\left(P_{t}\right)$ distribution of the parent mesons belonging to the cascade. By the time they reach the detector depth, however, their lateral spread about the common axis may be several meters. The spatial separation of any pair of muons in a bundle depends on the energy and interaction height of the parent cosmic ray, their relative $P_{t}$, the multiple Coulomb scattering of muons in the rock overburden above the underground laboratory, and, for oppositely charged particles, the geomagnetic field. These processes are essentially stochastic, and they contribute to a broadened muon lateral distribution. In some cases, these factors compete. For example, the hadronic interaction cross section increases generally as a function of $\ln (s)[3,4](\sqrt{s}=$ center-of-mass energy $)$, so that the interaction height in the exponential atmosphere increases slowly as the primary energy increases. But the opening angle of the mesons decreases roughly in proportion to the energy, which more than offsets the effect of the greater path length to the detector. However, when the incident particle is a heavy nucleus (with the same total energy as a single proton), the larger cross section induces an earlier interaction without the accompanying decrease in opening angle, so that the resultant muons tend to arrive at the detector with a larger separation.

Because of its large acceptance for downward muons $\left(\sim 3000 \mathrm{~m}^{2}\right.$ sr for the completed six supermodules) and excellent tracking $(\sim 1 \mathrm{~cm}$ average spatial resolution), MACRO [5] is well suited for the measurement of the muon lateral distribution. An underground muon detector typically measures the flux of muon pairs as a function of their separation. In the literature this measurement is often expressed in the form of a decoherence function which is strictly related to the muon pair distribution with respect to the shower axis. We therefore adopt the definition of the decoherence function [6] as the rate of muon pairs per unit area, per steradian, per pair separation determined on a plane orthogonal to the pair direction:

$$
G(r)=\frac{1}{\Omega T} \int \frac{d^{2} N_{p}(r, \theta, \phi) / d r d \Omega}{A(\theta, \phi)} d \Omega,
$$

where $d^{2} N_{p} / d r d \Omega$ is the density of pairs at distance $r$ and incidence angle $(\theta, \phi), A$ is the projected detector area in the $(\theta, \phi)$ direction, $\Omega$ is the total solid angle defined by the limits of integration, and $T$ is the exposure time.

The distribution measured by a finite-size detector is biased by the detector's dimensions. Pair separations that exceed the detector's dimensions clearly cannot be measured, nor can separations less than the spatial resolution. The average separation of the muon pairs exceeds $6 \mathrm{~m}$ at Gran Sasso Laboratory depths and the latter case includes less than $0.7 \%$ of the events, which is quite negligible in MACRO.

A detector-independent decoherence function can be unfolded from the measured one, up to the maximum pair distance allowed by the apparatus, provided that the detector geometry and efficiency are properly considered in track reconstruction of the penetrating particles.

Because the detector response characteristics heavily affect the measured pair distance distribution, only a detector-independent treatment of the data can provide results comparable with any detector-independent Monte Carlo prediction and/or other experimental data. In this respect we believe that it is important to establish crosschecking procedures to avoid any bias in the results. Two different and independent analysis methods have been used to unfold the detector-independent decoherence function from the measured lateral separations. These are described in Sec. IV below.

The decoherence function depends on the chemical composition of the primaries. However, this dependence turns out to be weak because at least $65 \%$ (in the case of the very extreme "heavy" composition model) of the multiple-muon data used to determine the function derives from a region of the cosmic-ray spectrum under 
$800 \mathrm{TeV}$, where relatively few heavy nuclei have sufficient energy to yield multiple muons in our detector [2].

We note that in the primary composition studies based upon the rates of multiple muons underground [2,7], the simulation of the muon multiplicity distribution in a finite-size detector is influenced by the knowledge of the muon lateral distribution. A comparison of the measured, detector-independent decoherence curve with the Monte-Carlo-generated curves used in the multiplicity analysis provides an important consistency check on the hadronic interaction processes employed in the simulations.

\section{MONTE CARLO SIMULATIONS}

The specific problem of determining the muon yield and lateral distribution deep underground, in the context of the general problem of describing the hadronic interaction and extensive air shower cascade, has been studied by different workers by means of Monte Carlo simulations. These simulations typically include an interaction model of primary nuclei with air nuclei, the cascade development, and the muon transport through the rock. In our Monte Carlo simulation, we used a parametrization obtained from the HEMAS (hadrons, electrons, and muons in air showers) code [8]. This code contains an interaction model founded upon a phenomenological description of minimum-bias events observed at $p p$ and $p \bar{p}$ collider energies [3], essentially in the central pseudorapidity $(\eta<3)$ region. This model, which includes observed scaling violations and transverse momentum correlation with the center-of-mass energy, is extrapolated to higher energy according to a $\ln ^{2} s$ behavior and to the high-rapidity (fragmentation) region. Nuclear target effects are included in order to reproduce the changes, with respect to hadron-hadron interactions, in multiplicity, transverse momentum, and rapidity that are observed in fixedtarget, hadron-nucleus experiments (mostly at $300 \mathrm{GeV} / c$ laboratory momentum) [9]. These features are assumed to be still valid at the higher energies relevant to underground experiments such as MACRO.

In the framework of the HEMAS code, nucleus-nucleus interactions can be treated in two different ways: (i) by superposition, where the interaction of a projectile of mass number $A$ and energy $E$ is treated as a beam of $A$ independently interacting nucleons with individual energies $E / A$; (ii) according to a model of nuclear fragmentation, as obtained from the analysis of a set of experimental data of primary nuclei interacting in nuclear emulsions [10].

The authors of HEMAS code estimate that the choice of one model with respect to the other renders a maximum variation of about $20 \%$ in the muon yield underground and in the average separation of muon pairs. We regard this variation as a measure of the systematic uncertainty introduced by the hadronic interaction model into the Monte Carlo program. Overall, HEMAS is considered a substantial improvement over previous efforts [1], when data from the CERN Sp $\bar{p}$ S and Fermilab Tevatron colliders were not yet available.

Unlike the codes used to simulate accelerator physics, in which the spectra of incident particles are nearly mononenergetic, the codes used to model high-energy cosmic-ray interactions must sample the projectile energy from a wide, steeply falling, continuous spectrum. This spectrum has a power-law behavior, and given the MACRO size, exposure time, and depth, several decades of energy must be sampled. The lower end of the relevant spectrum is set by the minimum overburden and corresponds to a primary threshold of a few TeV. The steepness of the spectrum sets a practical upper limit, which is effectively determined by the necessary exposure (acceptance $\times$ live time) required to detect a statistically significant number of high-energy events. The energy range in our simulations extends from 3 up to $10^{5} \mathrm{TeV}$.

We have investigated with HEMAS the range of kinematic variables, specifically primary energy and rapidity, accessible with multiple muon detected underground at the Gran Sasso Laboratory. As an input for the chemical composition of the primary cosmic rays, we have chosen the constant-mass-composition (CMC) model [11]. Figure 1 shows the energy distribution (in the nucleonnucleon system) for the interactions yielding multiplemuon events underground. The average c.m. energy for producing two or more muons is $\sim 515 \mathrm{GeV}$. Figure 2 shows the rapidity $(y)$ distribution of parent mesons yielding multiple muons underground in the nucleon-nucleon c.m. frame. About $30 \%$ of these mesons are produced at $y>5$. These values show that most of the relevant interactions of the primary-cosmic-ray nucleons occur, if not in the same rapidity range, on an energy scale covered by existing data from accelerators.

For the nucleus-nucleus system, the energy distribution is shown in Fig. 3. No accelerator data exist at present in this range as far as hadron-nucleus and nucleus-nucleus interactions are concerned.

The HEMAS Monte Carlo calculation produces lateral and multiplicity distributions of deep underground muons for each setting of mass of parent primary nucleus, its energy, its zenith angle, and rock depth. For convenience, these have been parametrized and used in

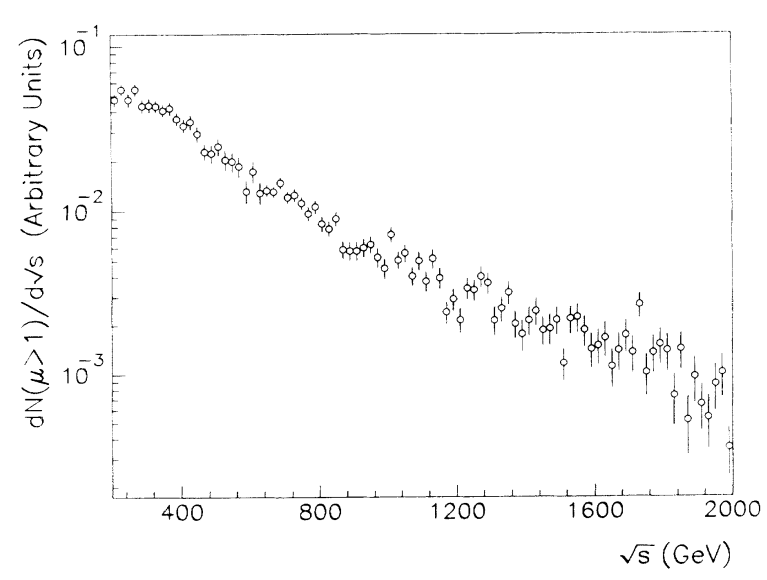

FIG. 1. Distribution of the muon bundles $\left(n_{\mu} \geq 2\right)$ vs the nucleon-nucleon center-of-mass energy $(\sqrt{s})$ of the primary interactions, as given by the HEMAS code using the CMC model. 


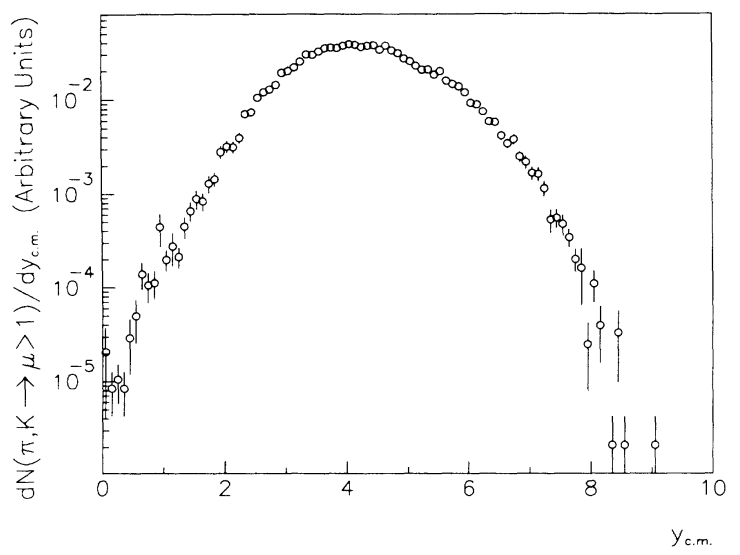

FIG. 2. Distribution of the muon bundles $\left(n_{\mu} \geq 2\right)$ vs the center-of-mass rapidity $\left(y_{\text {c.m. }}\right)$ of the secondary mesons produced in the primary-air interactions, as given by the HEMAS code using the CMC model.

the comparison of our data with the Monte Carlo predictions.

\section{EXPERIMENTAL CONFIGURATION}

The MACRO detector is located in Hall B of the Gran Sasso underground laboratory. Detailed descriptions of the apparatus are give in [5]. Briefly, it is a large-area detector equipped with streamer tube chambers, liquid scintillator tanks, and track-etch detectors arranged in a modular structure (supermodules). Each of the six supermodules is $12 \mathrm{~m} \times 12 \mathrm{~m} \times 9 \mathrm{~m}$ and consists of a $4.8-\mathrm{m}$ high lower level filled with rock absorber and a $4.2-\mathrm{m}$ high hollow upper level. In this paper only data from the lower level of the apparatus are included, and so only this level will be described further. The tracking is performed with the streamer tubes, which are distributed on ten horizontal planes, separated by $\sim 60 \mathrm{~g} \mathrm{~cm}^{-2}$ of $\mathrm{CaCO}_{3}$ rock absorbers, and on six planes on each vertical wall. The streamer tubes have a square cross section of $3 \times 3 \mathrm{~cm}^{2}$

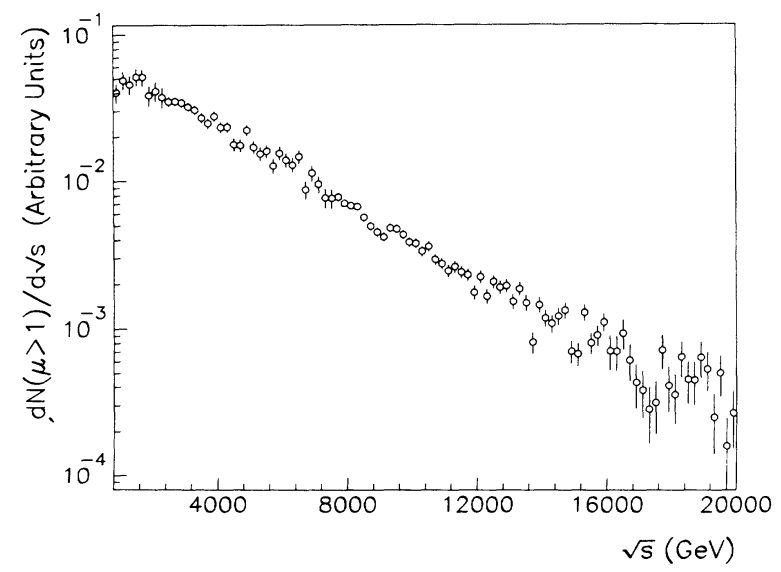

FIG. 3. Distribution of the muon bundles $\left(n_{\mu} \geq 2\right)$ as function of the nucleus-nucleus center-of-mass energy $(\sqrt{s})$ of the primary interaction, as given by the HEMAS code using the CMC model. and are $12 \mathrm{~m}$ long. For each plane two coordinates are digitally read out, the wire view and the pickup strip view. Pickup aluminum strips are $3 \mathrm{~cm}$ wide and are aligned at a stereo angle of $26.5^{\circ}$ with respect to the streamer tubes. This arrangement allows a spatial resolution of $1.1 \mathrm{~cm}$ in both views, corresponding to an intrinsic angular resolution of $0.2^{\circ}$ for muons crossing ten horizontal planes. Tracks on the different views can be associated in space in the majority of events, depending upon the spatial separation and multiplicity. This is automatically achieved when two tracks pass through separate detector modules. When they are in the same module, matching of hit wires and strips on the same detector plane is accomplished by taking advantage of the stereo angle of the strips with respect to the wires. In a fraction of cases, the track pattern correspondence between the two views is also used. The unique association of the tracks permits the reconstruction of the distance between muons starting from their projective views.

\section{DATA SELECTION}

The modularity of the apparatus allows us to collect data while we progressively enlarge the detector. The analysis presented here is based on events acquired with one supermodule (1SM) with $4900 \mathrm{~h}$ live time and two supermodules (2SM) with $2300 \mathrm{~h}$. About 48000 multiplemuon events have been collected in these periods. Multiple-muon events are $3.8 \%$ of total muon events in $1 \mathrm{SM}$ and $4.6 \%$ in $2 \mathrm{SM}$. The analysis of muon pair separation has been restricted to events with zenith angle not greater than $60^{\circ}$, to be consistent with the muon sample generated by the Monte Carlo program [8], which assumes a geometrically flat Earth and atmosphere. Secondary particles (e.g., $\delta$ rays, pions) produced by interactions in the surrounding rock or in the detector absorber are rejected by parallelism cut. This cut selects muon pairs with relative angle less than $3^{\circ}$ in the projected view. This criterion is very loose for the muon bundles at our depths, which have angular divergences around $1^{\circ}$ mainly because of the multiple scattering in the rock. In Table I we list the number of events and muon pairs that have been used in the analysis. The resulting total number of unambiguously reconstructed muon pairs is 20454 for 1SM and 31409 for the 2SM sample. In Table II is reported the fraction of unambiguously reconstructed muon pairs in events of different muon multiplicities and in the two different detector acceptances.

Figure 4 shows the distributions of the pair distances for each data sample, normalized to the total number of events falling in the range between 0 and $4 \mathrm{~m}$, namely, in the region of the maximum number of events. The distributions differ in the region of higher separations, reflecting the doubling of detector area.

\section{DETECTOR-INDEPENDENT ANALYSIS METHODS}

For a given event configuration, the containment efficiency, i.e., the fraction of the muon bundle that passes through the detector, depends on the apparatus acceptance. Furthermore, in a projective detector such 
TABLE I. Event sampling.

1SM: $4934.9 \mathrm{~h}$ live time (Feb. 1989-Apr. 1990)

Total number of multiple muon events $\quad 22151$

Number of events with at least one unambiguously reconstructed pair $\quad 16432$

Number of events surviving the $\theta<60^{\circ}$ cut $\quad 15972$

Number of unambiguously reconstructed pairs $\quad 21418$

Number of unambiguously reconstructed pairs surviving parallelism cut $\quad 20454$

2SM: 2334.3 h live time (May 1990-Sept. 1990)

Total number of multiple muon events 26016

Number of events with at least one unambiguously reconstructed pair $\quad 21696$

$\begin{array}{ll}\text { Number of events surviving the } \theta<60^{\circ} \text { cut } & 21120\end{array}$

Number of unambiguously reconstructed pairs $\quad 31829$

\begin{tabular}{lr} 
Number of unambiguously reconstructed pairs surviving parallelism cut & 31409 \\
\hline
\end{tabular}

MACRO, we must calculate the percentage of events for which it is possible to measure the actual muon pair separation (unambiguously reconstructed pairs), i.e., the fraction of pairs whose projected tracks on wire and strip views can be unambiguously associated one to the other. The probability to reconstruct completely one pair depends on muon separation, direction, and the detector geometry.

As already mentioned, two independent methods have been used to perform the unfolding of the decoherence function from the experimental data. Both account for the finite size of the detector and its efficiency for reconstructing muon distances.

The first unfolding procedure calculates the containment and reconstruction efficiency of each muon pair as a function of the incidence angle and pair distance, by means of a detailed Monte Carlo simulation of the experimental configuration. In this procedure we apply the weight $2 / M(M-1)$ to the independent pairs belonging to the same event and we follow the same prescription for the simulated data set, considering at this time the actual event multiplicity $N$. In this way the total weight of each event is the same (i.e., weight $=1$ ), so that rarer highmultiplicity events do not bias the distributions. We emphasize that this detector-independent decoherence function, hereafter named $G^{\prime}(r)$, with respect to the distribution containing all the independent pairs, receives a smaller contribution from the richest bundles, and for this reason it reflects the lower-primary-energy region to which MACRO is sensitive.

The second unfolding procedure is based on a Monte Carlo treatment of experimentally observed events. It allows us to estimate the contribution of a bundle whose true multiplicity is $N$ to events whose detected multiplicity is $M$. It is thus possible to give a decoherence function $G_{N}(r)$ for each true bundle multiplicity. This decoherence can be compared directly with the distributions obtained by a detector-independent Monte Carlo program. Because of the statistical limitations of the event sample considered in this analysis, only the decoherence func-

TABLE II. Efficiency of unambiguous reconstruction of the pairs vs muon multiplicities.

\begin{tabular}{crrrr}
\hline \hline & \multicolumn{2}{c}{ 1SM } & $\begin{array}{c}\text { Number of } \\
\text { unambiguously }\end{array}$ \\
Muon multiplicity & Number of events & Number of pairs & reconstructed pairs & $(\%)$ \\
\hline 2 & 13734 & 13734 & 13016 & 95 \\
3 & 1472 & 4416 & 4302 & 97 \\
4 & 502 & 3012 & 1728 & 57 \\
5 & 167 & 1670 & 738 & 44 \\
6 & 51 & 765 & 267 & 35 \\
7 & 27 & 567 & 223 & 39 \\
8 & 13 & 364 & 133 & 37 \\
9 & 5 & 180 & 41 & 23 \\
10 & 1 & 45 & 6 & 13 \\
& & & & 98 \\
2 & 17 & 17424 & 6831 & 98 \\
3 & 2314 & 6942 & 3400 & 66 \\
4 & 853 & 5118 & 1815 & 60 \\
5 & 301 & 3010 & 1163 & 57 \\
6 & 136 & 2040 & 603 & 52 \\
7 & 55 & 1155 & 301 & 41 \\
8 & 26 & 728 & 84 & 39 \\
9 & 6 & 216 & 13 & 14 \\
10 & 2 & 90 & & 98 \\
\hline
\end{tabular}




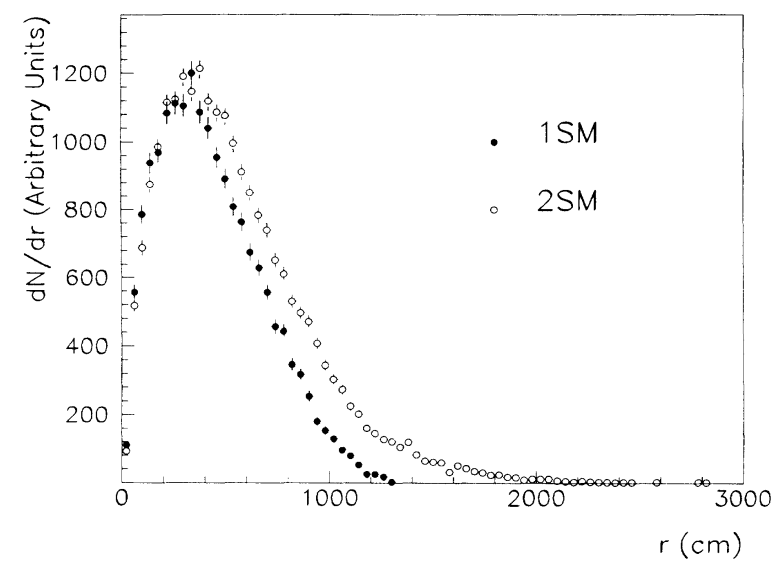

FIG. 4. Muon separation distributions measured by MACRO with one supermodule $\left(144 \mathrm{~m}^{2}\right.$ horizontal area) and two supermodules ( $288 \mathrm{~m}^{2}$ horizontal area).

tions at the lowest multiplicity (i.e., dimuon, trimuon, and quadmuon events) have been analyzed.

The agreement between the two methods constitutes an important test for the reliability of the detectorindependent decoherence function.

\section{A. Unfolding of the decoherence from the whole pair sample}

The first unfolding procedure consists in determining, for each detector configuration (1SM, 2SM), the containment probability $\boldsymbol{P}_{c}(r, \theta, \phi)$ as a function of the muon separation $r$ and of the incidence angle $(\theta, \phi)$. This probability vanishes as $r$ approaches the maximum detector size. The efficiency $\epsilon$ for measuring the separation of a muon pair in MACRO is obtained as the product of $P_{c}$ times the efficiency to uniquely determine the pair distance, $E_{\text {ass }}(r, \theta, \phi)$.

In practice, $\epsilon$ is obtained by means of a detailed Monte Carlo program based on the GEANT [12] code simulating the MACRO detector. We have generated $N_{g}$ muon pairs at fixed relative separation $r$ and fixed incidence angle in which at least one muon falls inside the apparatus. We have recorded the number of pairs entering the detector, $N_{\text {pair }}$, and the number of unambiguously reconstructed muon paris, $N_{\text {recon }}$ using the same programs employed to analyze the real data. The Monte Carlo data sets refer to steps of $40 \mathrm{~cm}$ in distance and $0.06 \mathrm{sr}$ in solid angle. The steps are small enough to have little variation in detector acceptance and efficiency, and they contain around 1000 events to mitigate statistical fluctuations. We obtain the efficiency from

$$
\epsilon(r, \theta, \phi)=N_{\text {recon }}(r, \theta, \phi) / N_{g}(r, \theta, \phi)
$$

and, separately,

$$
E_{\text {ass }}(r, \theta, \phi)=N_{\text {recon }}(r, \theta, \phi) / N_{\text {pair }}(r, \theta, \phi),
$$

which is used by the second unfolding procedure (see Sec. V B). This calculation has been performed for one and two supermodules (horizontal planes only). The efficiency at any distance has been determined by spline interpolation of the values obtained from the Monte Carlo calculation.

We have

$$
\frac{d F(r, \theta, \phi)}{d \Omega} d \Omega
$$

$$
=\frac{2}{\Omega T \Delta r} \frac{\sum_{i=1}^{N_{b}(\theta, \phi)} N_{p}^{i}(r, \theta, \phi) /\left[M^{i}\left(M^{i}-1\right)\right]}{A(\theta, \phi) \epsilon(r, \theta, \phi)},
$$

and the decoherence function is finally given by

$$
G^{\prime}(r)=\int \frac{d F(r, \theta, \phi)}{d \Omega} d \Omega,
$$

where $N_{b}(\theta, \phi)$ is the number of collected bundles in the $(\theta, \phi)$ direction, $N_{p}^{i}(r, \theta, \phi)$ is the number of pairs belonging to the $i$ th bundle at separation $r, M^{i}$ is the number of unambiguously reconstructed pairs in the bundle, $A(\theta, \phi)$ is the projected detector area in a plane orthogonal to the $(\theta, \phi)$ direction, $\epsilon(r, \theta, \phi)$ is the efficiency function described above, $\Delta r$ is the separation bin width $(40 \mathrm{~cm}), \Omega$ is the solid angle defined by the limits of integration, $2 \pi\left[1-\cos \left(60^{\circ}\right)\right]$, and $T$ is the exposure time.

\section{B. Unfolding}

of the decoherence from the bundle configurations

In the second unfolding method, we calculate the acceptance of different multiplicity muon bundles from their geometrical configuration and incidence direction with respect to the detector. For each detected event, a geometrical configuration $C$ is defined by the positions of the muons with respect to the axis of the bundle in the plane orthogonal to the incidence direction $(\theta, \phi)$. To define the configuration $C$, the muon bundle must be unambiguously reconstructed. Therefore only bundles with projected tracks unambiguously associated with one another can be handled by this technique. This bias affecting the event sample has been considered in the analysis as shown below.

The method proceeds through the following steps: (a) For each event with multiplicity $M$ and direction $(\theta, \phi)$, its configuration $C$ is randomly rotated and translated $N_{\text {try }}$ times over an area $A_{\text {gen }}$ larger than the detector's projected area $A_{\text {det }}$, producing $N_{\text {try }}$ "pseudo events"; (b) For each simulated "pseudoevent" the multiplicity $M^{\prime}$ $(\leq M)$ is computed using the same criteria employed to analyze real data; and, (c) the acceptance to detect that event as a bundle with multiplicity $M^{\prime} \leq M$ is then defined by

$$
A\left(M \rightarrow M^{\prime}, C, \theta, \phi\right)=\lim _{N_{\mathrm{try}} \rightarrow \infty} A_{\mathrm{gen}} \frac{n\left(M^{\prime}\right)}{N_{\mathrm{try}}},
$$

where $n\left(M^{\prime}\right)$ is the number of "pseudoevents" detected with multiplicity $M^{\prime}$. In practice, we choose $N_{\text {try }}=10000$, which is a good compromise between sufficient statistical accuracy on the calculated acceptance and a reasonable computing time.

To evaluate the decoherence function for a given true 
multiplicity $N$, we analyze the events with multiplicity $\geq N$. We calculate the decoherence function by weighting each detected event with the inverse of its relevant acceptance, $A(N \rightarrow N)$, and then we take into account the contributions of events with true multiplicity $N_{0}>N$ which are detected as bundles with multiplicity $N$. This contribution is estimated from events with detected multiplicity $N_{0}>N$ and from the calculated acceptances $A\left(N_{0}>N \rightarrow N\right)$. The bias introduced by the requirement for unambiguous association is taken into account by weighting each pair in the event with the inverse of the efficiency of unambiguous pair reconstruction, $E_{\text {ass }}(r, \theta, \phi)$. This efficiency is a function of the pair separation and incidence direction.

For each true multiplicity $N$, we obtain

$$
\begin{aligned}
\frac{d F_{N}(r, \theta, \phi)}{d \Omega} d \Omega= & \frac{1}{\Omega T \Delta r E_{\mathrm{ass}}(r, \theta, \phi)} \\
& \times \sum_{i=1}^{N_{b}(N, \theta, \phi)} \frac{N_{p}^{i}(r, \theta, \phi)}{A^{i}(N \rightarrow N, C, \theta, \phi)} \\
& \quad \times\left[1-g\left(N_{0}>N \rightarrow N\right)\right] .
\end{aligned}
$$

The decoherence function is then given by

$$
G_{N}(r)=\int \frac{d F_{N}(r, \theta, \phi)}{d \Omega} d \Omega,
$$

where $N_{b}(N, \theta, \phi)$ is the number of completely reconstructed bundles with multiplicity $N$ and incidence direction $(\theta, \phi), N_{p}^{i}$ is the number of pairs belonging to the $i$ th bundle at separation $r, A^{i}$ is the acceptance defined above, $E_{\text {ass }}(r, \theta, \phi)$ is the association efficiency, $g\left(N_{0}>N \rightarrow N\right)$ is the estimated fraction of events with true multiplicity $N_{0}>N$ detected as bundles with multiplicity $N, \Delta r$ is the separation bin width $(40 \mathrm{~cm}), \Omega$ is the total solid angle defined by the limits of integrations, $2 \pi\left[1-\cos \left(60^{\circ}\right)\right]$, and, $T$ is the exposure time.

We have found that, at least for low multiplicities, the correction term $(1-g)$ is dominated by the bundles with

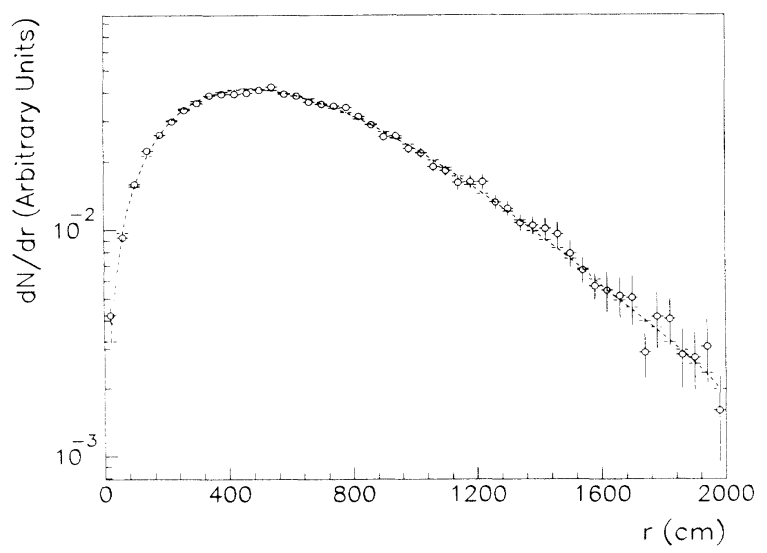

FIG. 5. Comparison of the input distance distribution and the reconstructed function using the first unfolding method (see Sec. V A). Dotted line, input function; points with error bars, results of the unfolding procedure.

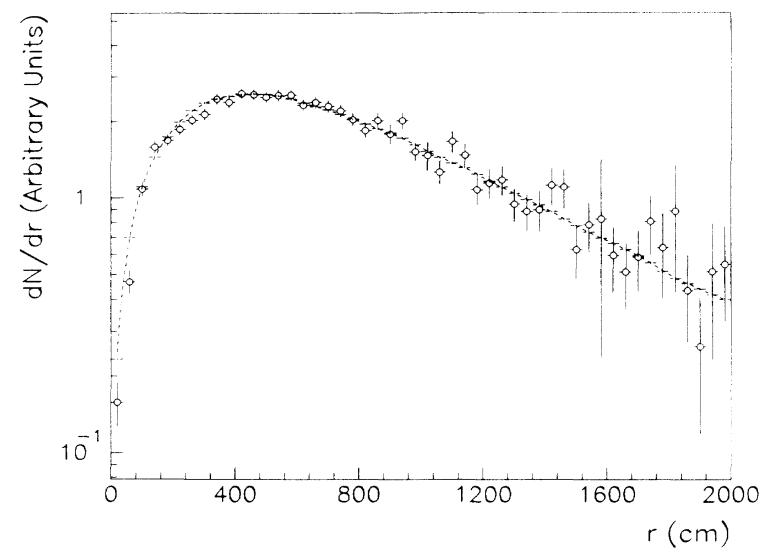

FIG. 6. Comparison of the input distance distribution and the reconstructed function using the second unfolding method (see Sec. V B). Dotted line, input function; points with error bars, results of the unfolding procedure.

multiplicity $N+1$ and is simply a scale factor, independent from $(r, \theta, \phi)$.

\section{Consistency check of the two methods}

In order to check the two unfolding procedures, we tried to reconstruct an a priori known muon lateral distribution which spans the entire distance range of real pairs. The events generated by this distribution have been processed through the same unfolding procedures as experimental data. The agreement between the unfolded curves and the input function (Figs. 5 and 6) confirms the ability of our algorithms to reconstruct the decoherence distribution.

Both procedures provide compatible decoherence functions when data acquired from one and two supermodules are analyzed. Figure 7 shows the superposition of these decoherence curves, normalized to the total number of events that fall within the common separation interval $(12 \mathrm{~m})$. The consistency of the results constitutes another check for the analysis methods and allows us to combine the entire analyzed data sample. Furthermore, because

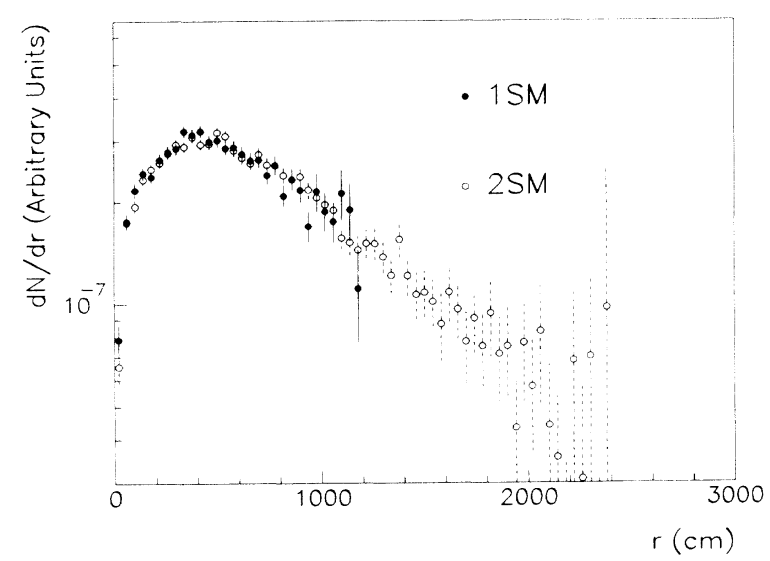

FIG. 7. Comparison of the decoherence functions for one (solid circles) and two (open circles) supermodules. 
the results of each method mutually agree, we infer that the procedures properly account for the detector performance, within present statistics.

\section{RESULTS AND COMPARISON WITH MONTE CARLO DATA}

The shape of the decoherence function has been compared with the results of the Monte Carlo simulation in Sec. II, based on the HEMAS code published in [8]. Primary nuclei have been sampled from an isotropic angular distribution, in the energy range from 3 up to $10^{5} \mathrm{TeV}$, and divided into five contiguous energy bands. From each band similar event statistics have been compiled. A total of about $8 \times 10^{5}$ underground muons have been generated for each chemical composition model of the primary cosmic rays.

The simulation code does not include geomagnetic-field effects. We have independently evaluated the average muon separation induced by the magnetic field and found it to be approximately $0.5 \mathrm{~m}$. This is smaller than the systematic uncertainty introduced by the treatment of the nucleus-nucleus interaction in the Monte Carlo code, which is about $20 \%$ of the average lateral displacement of muons.

Another systematic uncertainty is introduced by our assumed model of mass composition of the cosmic-ray primaries. We have used two composition models in addition to the CMC to investigate this point. These are the Maryland composition [13] ("heavy"), which is heavier than the CMC, and a low-energy composition (LEC) with an enhanced proton component [14] ("light"), which is lighter than the CMC. All models have been adjusted to the same all-particle spectrum [15]. The decoherence functions related to different mass compositions are shown in Fig. 8. The average separation changes by about $10 \%$ between the two extreme models, going from $883 \mathrm{~cm}$ ("heavy" model) to $806 \mathrm{~cm}$ ("light" model). This difference is within the expected systematics of the interaction model employed to simulate the atmospheric showers. Conversely, the difference in muon multiplicity rates predicted by these models can be quite well ex-

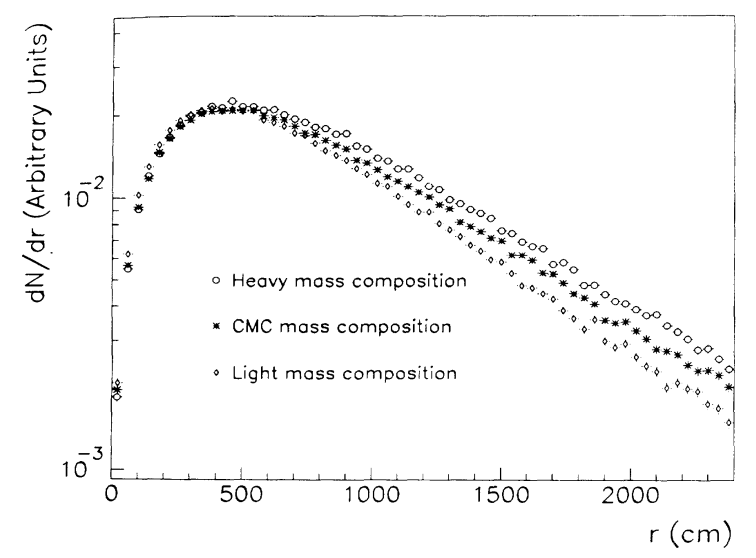

FIG. 8. Simulated decoherence functions for three different mass composition models.

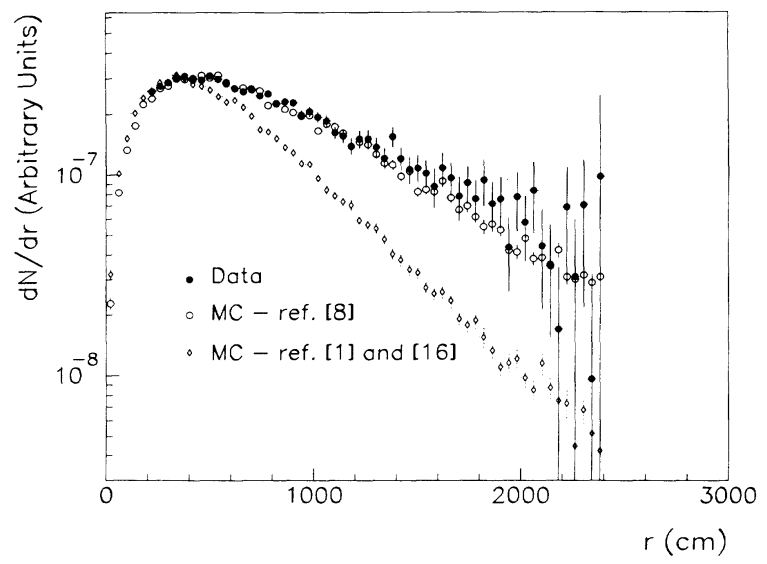

FIG. 9. Comparison of the experimental decoherence distribution with simulations from the parametrization of two different Monte Carlo codes, using the CMC model.

plored by our experiment and it is the subject of another paper [2].

Figure 9 shows the experimental decoherence curve (average of 1SM and 2SM samples) obtained by our first unfolding procedure superimposed with the results of this Monte Carlo calculation, assuming the CMC model as the primary mass composition [11]. In the same figure, we also show the results obtained by replacing in the Monte Carlo simulation the parametrization of the shower development with that published in $[1,16]$, where a simplified hadronic interaction model was used. Such a model is evidently not consistent with our data. In Fig. 9 we also note a possible deviation at large separations between data and the HEMAS model, but this is within the systematic uncertainty introduced by the hadronic interaction model. In Figs. 10(a) and 10(b), we report analogous comparisons for dimuon and trimuon bundles in which our second unfolding procedure is used.

The muon separation depends mainly on the muon production height, transverse momentum of parent mesons, and multiple scattering of the muons in the rock

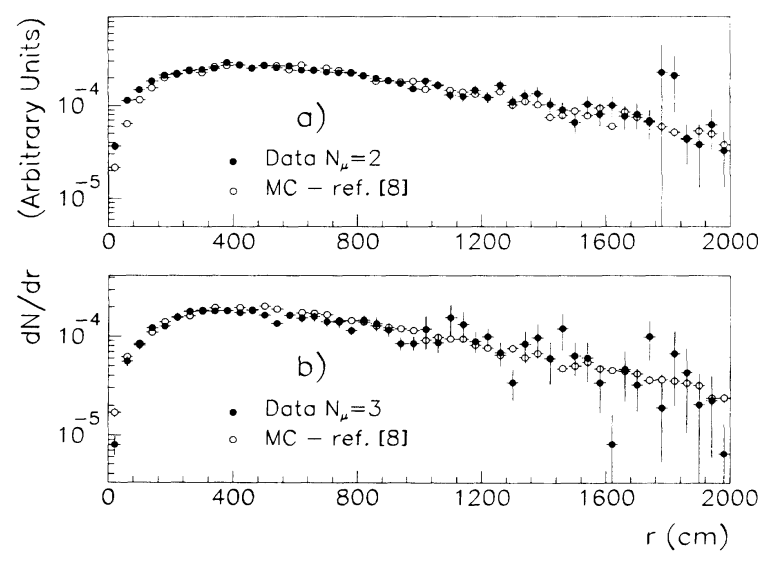

FIG. 10. Comparison of the experimental decoherence distribution for (a) the dimuon sample and (b) the trimuon sample with the simulation performed using the parametrization of Ref. [8]. 


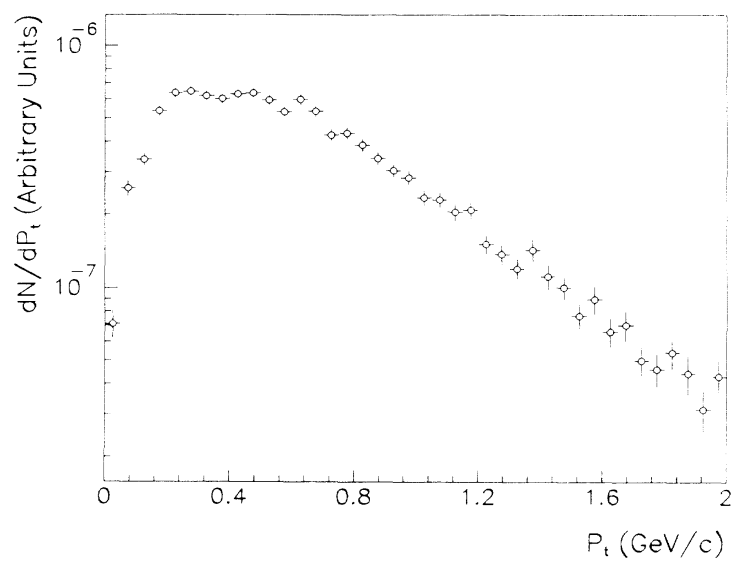

FIG. 11. Distribution of the muon bundles $\left(n_{\mu} \geq 2\right)$ vs the transverse momentum of the parent mesons, as given by the HEMAS code using the CMC model.

surrounding the laboratory. We computed with the HEMAS code these quantities. In Fig. 11 we show the distribution of the transverse momentum of the parent mesons giving at least two muons underground. The average value is $550 \mathrm{MeV} / \mathrm{c}$. In Figs. 12(a) and 12(b), we show the average muon displacement from the shower axis as a function of the transverse momentum and production height, measured along the shower axis. The average vertical production height is $20 \mathrm{~km}$, and the average displacement from the shower axis due to the multiple scattering through the rock overburden is $1.2 \mathrm{~m}$.

In order to study the decoherence function's behavior at different muon average energies in the atmosphere (reflecting different average primary energies) and at different mean distances from the primary interaction point, we integrate over restricted zenith-angle intervals and over specific windows of rock depth. In this analysis we must avoid correlations between rock depth and angle that can be introduced by the mountain shape. To study the dependence on the zenith angle, we have chosen a depth interval ranging from 3750 up to $4150 \mathrm{hg} / \mathrm{cm}^{2}$,

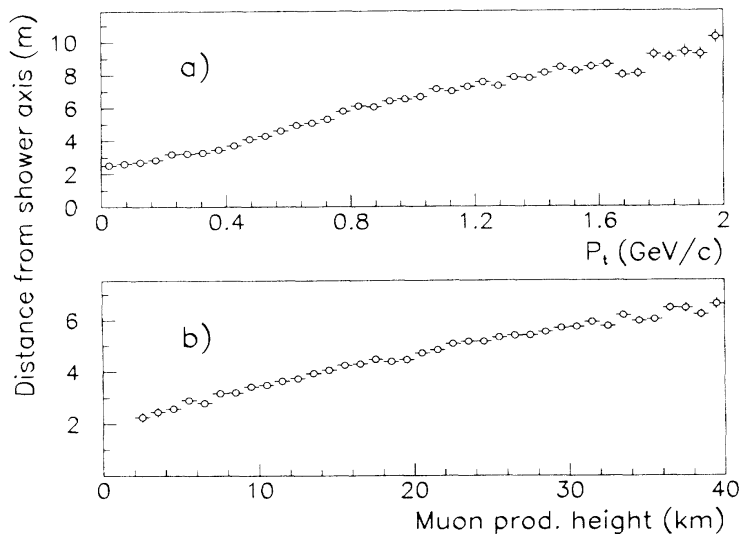

FIG. 12. Average distance from the shower axis for muon bundles detected underground (a) vs the transverse momentum of parent mesons and (b) vs the muon production height measured along the shower axis.

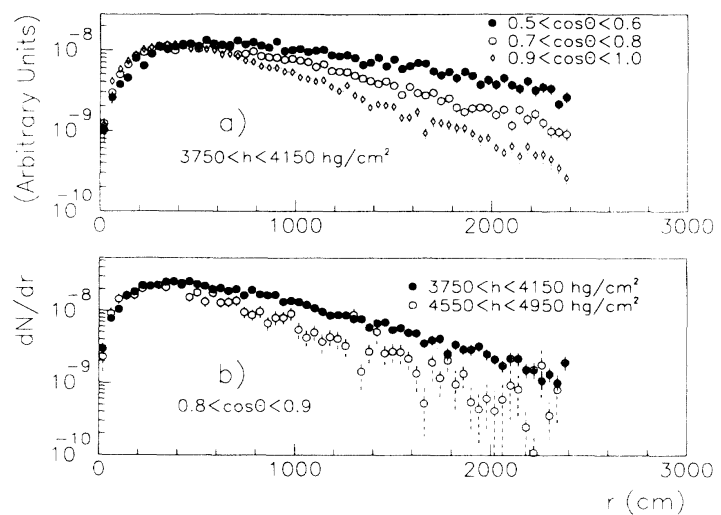

FIG. 13. Simulated decoherence function in a fixed rock depth interval (a) for three different zenith ranges and (b) in a fixed zenith-angle interval for two different ranges of rock depth.

where all $\cos \theta$ intervals give comparable contribution to the event sample. To investigate the rock depth dependence, a suitable $\cos \theta$ interval is that between 0.8 and 0.9 , for which we can study the rock depths from 3350 up to $4950 \mathrm{hg} / \mathrm{cm}^{2}$. We have subdivided this interval into four regions $400 \mathrm{hg} / \mathrm{cm}^{2}$ wide.

Figure 13(a) shows the Monte Carlo results according to Ref. [8] for three different $\cos \theta$ windows at fixed depths, and Fig. 13(b) is at fixed $\cos \theta$ for two depth intervals. We see that the average separation increases with zenith angle and decreases with rock depth. In Fig. 14 are shown the experimental and Monte Carlo data at $0.8<\cos \theta<0.9$ and $3750<h<4150 \mathrm{hg} / \mathrm{cm}^{2}$. In Table III we list the average distances, computed for experimental and Monte Carlo (MC) data between 0 and $24 \mathrm{~m}$, obtained from decoherence functions at various depths and zenith angles. It is important to note that the behavior predicted by the simulation is quantitatively confirmed.

Other experiments have made similar measurements. The Fréjus Collaboration has performed a detectorindependent measurement of the lateral displacement of

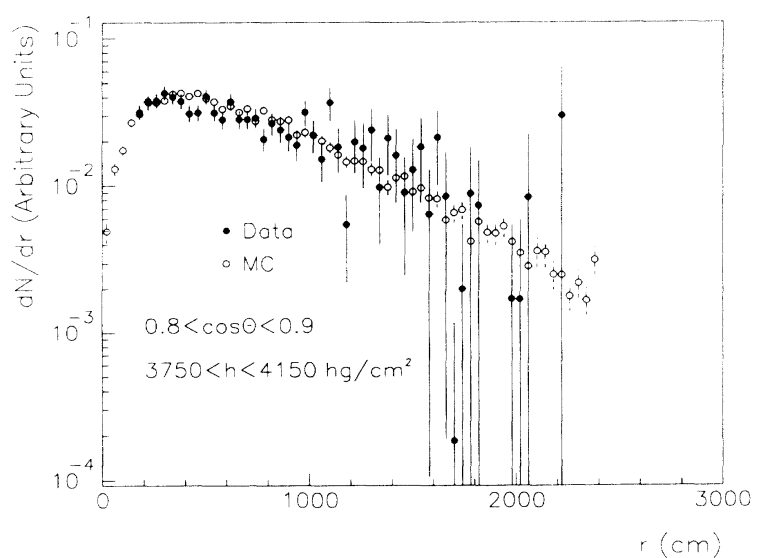

FIG. 14. Comparison of the experimental decoherence distribution obtained by the first unfolding method with the Monte Carlo results, for a given zenith angle and rock depth interval. 
TABLE III. Mean distances (0-24 m).

\begin{tabular}{ccc}
\hline \multicolumn{3}{c}{$3570<h<4150 \mathrm{hg} / \mathrm{cm}^{2}$} \\
$\cos \theta$ & $1877 \pm 400$ & $\langle r\rangle(\mathrm{cm}) \mathrm{MC}$ \\
\hline $0.5-0.6$ & $955 \pm 150$ & $1021 \pm 7$ \\
$0.6-0.7$ & $851 \pm 100$ & $927 \pm 5$ \\
$0.7-0.8$ & $786 \pm 50$ & $843 \pm 5$ \\
$0.8-0.9$ & $667 \pm 30$ & $765 \pm 6$ \\
$0.9-1.0$ & $0.8<\cos \theta<0.9$ & $699 \pm 4$ \\
& $\langle r\rangle(\mathrm{cm})$ data & $\langle r\rangle(\mathrm{cm}) \mathrm{MC}$ \\
Rock depth $\left(\mathrm{hg} / \mathrm{cm}^{2}\right)$ & $801 \pm 30$ & $831 \pm 4$ \\
\hline $3350-3750$ & $786 \pm 50$ & $765 \pm 6$ \\
$3750-4150$ & $785 \pm 80$ & $675 \pm 9$ \\
$4150-4550$ & $725 \pm 90$ & $619 \pm 13$ \\
$4550-4950$ &
\end{tabular}

muons from the shower axis [17], based on an assumption of the precise functional form of lateral displacement distribution. They find a correlation of the decoherence with zenith angle but not with rock depth. A decrease of the average displacement for increasing muon multiplicity is also found, as expected from the correlation of multiplicity with primary energy. Similar measurements have been performed by the Baksan experiment [18].

\section{CONCLUSIONS}

Our present analysis is consistent with the hadronic interaction model used in [8]. The decoherence function derives from the convolution of the height of primary interaction in the atmosphere with the $P_{t}$ of the parent mesons in the laboratory energy range $10-10^{3} \mathrm{TeV}$. An analysis based on a full Monte Carlo simulation of nucleus-nucleus interactions can provide a powerful tool to assess the validity of different models currently employed to describe the hadronic cascades. Much work is in progress in this field, but nevertheless our results already suggest that the $P_{t}$ distribution of mesons produced in the fragmentation region at $\sqrt{s} \sim 500 \mathrm{GeV}$ follows the distribution measured by collider experiments in the central rapidity region, provided that the extension from $p p$ results to $p N$ and $N N$ interactions described in [8] is still valid at these energies.

In the future new data will be available with the full MACRO length (over $70 \mathrm{~m}$ ). This will allow us to increase the sensitivity of our measurements and to better investigate the dependence of muon separation on the different parameters. Possible scaling violations in the fragmentation region due to parton-parton scattering could be revealed by the decoherence shape at large distances. We shall also be able to study the decoherence function with the sample of data taken in coincidence with the EASTOP surface array [7], which measures size of extensive air showers.
[1] T. K. Gaisser and T. Stanev, Nucl. Instrum. Methods A 235, 183 (1985).

[2] MACRO Collaboration, S. Ahlen et al., Phys. Rev. D 46, 895 (1992).

[3] UA5 Collaboration, G. J. Alner et al., Phys. Lett 160B, 199 (1985); 167B, 476 (1986).

[4] CDF Collaboration, S. White et al., in Proceedings of the Fourth International Conference on Elastic and Diffractive Scattering, La Biodola, Elba, Italy, edited by F. Cervelli and S. Zucchelli [Nucl. Phys. B (Proc. Suppl.) 25B, 19 (1992)].

[5] MACRO Collaboration, in Proceedings of the XXIst International Cosmic Ray Conference, Adelaide, Australia, 1989, edited by R. J. Protheroe (Graphic Services, Northfield, South Australia, 1990), Vol. 10, p. 256.

[6] P. Sokolsky, Introduction to UltraHigh Energy Cosmic Ray Physics (Addison-Wesley, Reading, MA, 1989).

[7] MACRO and EASTOP Collaborations, in Proceedings of the 22nd International Cosmic Ray Conference, Dublin, Ireland, 1991, edited by M. Cawley et al. (Dublin Institute for Advanced Studies, Dublin, 1992), Vol. 4, Article HE
4.2 and Vol. 2, Article OG 6.1.23.

[8] C. Forti et al., Phys. Rev. D 42, 3668 (1990).

[9] J. W. Cronin et al., Phys. Rev. D 11, 3105 (1975).

[10] P. S. Freyer and C. J. Waddington, in Cosmic Rays and Particle Fields - 1978 (Bartol Conference), Proceedings of the Bartol Conference on Cosmic Rays and Particle Physics, edited by T. K. Gaisser, AIP Conf. Proc. No. 49 (AIP, New York, 1979), p. 87.

[11] J. Kempa and J. Wdowczyk, J. Phys. G 9, 1271 (1983).

[12] R. Brun et al., Report No. CERN DD/EE/84-1, 1984 (unpublished).

[13] J. A. Goodman et al., Phys. Rev. Lett. 42, 854 (1979).

[14] C. Fichtel and J. Linsley, Astrophys. J. 300, 474 (1986).

[15] G. Auriemma et al., in Proceedings of the XXIst International Cosmic Ray Conference [5], Vol. 9, p. 362.

[16] G. Bologna et al., Nuovo Cimento C 8, 76 (1985).

[17] Ch. Berger et al., Phys. Rev. D 40, 2163 (1989).

[18] E. V. Budko et al., in, Proceedings of the XXth International Cosmic Ray Conference, Moscow, USSR, 1987 edited by V. A. Kozyarivsky et al. (Nauka, Moscow, USSR, 1987), Vol. 6, p. 221. 